\title{
La antropología redescubre la sexualidad: un comentario teórico*
}

\author{
Carole S. Vanee**
}

A pesar de la reputación de ser una disciplina abierta al estudio de la sexualidad, la antropologia ha apoyado su investigación con reticencias. Tanto la investigación como la teoria antropológica se han desarrollado lentamente, manteniendo un paradigma teórico estable (el modelo de influencia cultural) desde la década de los veinte hasta la de los noventa. A pesar de que la antropologia ha ido más allá de los esquemas deterministas y esencialistas que aún son comunes en la biomedicina, sus trabajos siguen visualizando aspectos importantes de la sexualidad como universales y transculturales.

La teoria de la construcción social ha ofrecido un desafio a los modelos antropológicos tradicionales y ha sido responsable del reciente surgimiento de trabajos innovadores sobre sexualidad, tanto en la antropología como en otras disciplinas, a partir de 1975. En este artículo se exploran las raices teóricas y las implicaciones de la teoría construccionista.

La creciente competencia entre el paradigma de influencia cultural y el paradigma construccionista ha sido alterada por el surgimiento del sida y el subsecuente incremento en los apoyos para la investigación de la sexualidad. Por un lado, el crecimiento de los recursos amenaza con fortalecer los modelos esencialistas en contextos biomédicos, asi como los modelos de influencia cultural dentro de la antropología. Por otro lado, la complejidad y ambigüedad inherentes al estudio de la sexualidad pueden revelar la solidez de las aproximaciones construccionistas, y estimular el desarrollo de la teoría y la investigación antropológica.

Palabras clave: antropología y sexualidad, teoría de la construcción social, sida y sexualidad.

* Este artículo fue traducido del inglés con el permiso de la autora. El original "Anthropology Rediscovers Sexuality: A Theoretical Comment", fue publicado en Social Science and Medicine, vol. 33, núm. 8, 1991, pp. 875-884. Por esta razón, el trabajo que aquí se publica no sigue fielmente las convenciones editoriales de la revista Estudios Demográficos y Urbanos.

Quiero dar gracias a Frances M. Doughty por su provechosa conversación, sus invaluables sugerencias editoriales y su generoso aliento. Agradezco los comentarios, la paciencia y el entusiasmo de Shirley Lindenbaum. Gracias también a Lisa Duggan, Gayle Rubin, David Schwartz, Gilbert Zicklin, Jonathan Katz, Janice Irvine, Ann Snitow, Nan Hunter, Jennifer Terry, Jacqueline Urla, Libbett Crandon, William Hawkeswood, Jeanne Bergman, Faye Ginsburg y a los anónimos revisores de Social Science and Medicine por sus comentarios. Gracias a Pamela Brown-Peterside por su ayuda en la investigación.

Este trabajo fue presentado en el panel "Anthropology Rediscovers Sex" del Encuentro Anual de la American Anthropological Association de 1988. Quiero agradecer a Shirley Lindenbaum, organizadora de dicho panel, así como a los participantes, por el vivo diálogo que se llevó a cabo. También me beneficié de los comentarios realizados por los miembros del Medical Anthropology Colloquium en la Universidad de Columbia. La responsabilidad por los puntos de vista expresados en este artículo sigue siendo mía.

** Division of Sociomedical Sciences, School of Public Health, Columbia University. 
En el principio estuvo el sexo y el sexo estará en el fin [...] Sostengo -y ésta es mi tesis-que el sexo es un rasgo del hombre y la sociedad que siempre fue central y lo sigue siendo...

GOLDENWEISER, 1929: 53

Esta sentencia introductoria del ensayo de Alexander Goldenweiser "Sexo y sociedad primitiva", sugiere que la sexualidad ha sido un foco importante en la investigación antropológica. Tal es la reputación que los antropólogos se han concedido a sí mismos: investigadores intrépidos de las costumbres sexuales a lo largo del mundo, y quebrantadores de los erofóbicos tabúes intelectuales, comunes en otras disciplinas más timoratas.

En realidad, las relaciones entre la antropología y el estudio de la sexualidad han sido más complejas y contradictorias. La antropología, como campo de estudio, ha estado lejos de ser valiente, o aun de portarse de manera adecuada, al estudiar la sexualidad (Fisher, 1980; Davis y Whitten, 1987). Más bien, la disciplina parece compartir con frecuencia las visiones culturales prevalecientes acerca de que la sexualidad no es un área de estudio enteramente legítima, y de que tal estudio arroja necesariamente sospechas, no sólo sobre la investigación, sino también sobre los motivos y el carácter del investigador. En esto no hemos sido peores, aunque tampoco mejores, que en otras disciplinas de las ciencias sociales.

Manifestaciones de esta actitud abundan en los cursos de posgrado y en las estructuras de reconocimiento de la profesión. Pocos departamentos de posgrado ofrecen programas para el estudio de la sexualidad humana. Como resultado, no hay canales construidos para transmitir el conocimiento antropológico sobre la sexualidad a la siguiente generación de estudiantes. La ausencia de una comunidad de académicos empeñados en la problemática de la sexualidad detiene efectivamente el avance del campo; los estudiantes interesados en el tema perciben que deben redescubrir el trabajo de generaciones anteriores a la suya. La mayoría de los asesores desalientan activamente a los estudiantes de posgrado para no seguir trabajos de campo o realizar tesis sobre sexualidad, por temor a que el tema ponga en riesgo su carrera. En el mejor de los casos, los estudiantes son exhortados a terminar sus doctorados, hacerse de credenciales y reputación y aun obtener prestigio, lo cual, se dice, los pondrá en una mejor situación para emprender el estudio de la sexualidad. En vez de redoblar el trabajo colectivo necesario para remediar esta grave limitación estructu- 
ral de nuestra disciplina, lo que se transmite a los estudiantes es un claro mensaje: la sexualidad es tan peligrosa en el terreno intelectual que puede arruinar la carrera de personas que, siguiendo otro tema, podrían ser estudiantes de posgrado y académicos competentes.

Tampoco hay alguna especialidad después de los estudios de posgrado para antropólogos interesados en la sexualidad. Como nunca alcanza el estatus de una especialización apropiada, la sexualidad se mantiene marginalmente. Es dificil encontrar recursos, pues las agencias siguen temiendo la potencial controversia pública sobre el tema. Los colegas suelen mostrarse suspicaces e hipercríticos ante el solo hecho de que la sexualidad sea tratada en términos escolares adecuados o legítimos. ${ }^{1}$ Los trabajos de campo raramente se enfocan directa o completamente sobre la sexualidad, si es que tocan el tema; más bien, los investigadores de campo recolectan ciertos datos como pueden, algunos de los cuales nunca publican por temor a dañar su reputación profesional. Algunos antropólogos se refugian en la sexología, quizás más hospitalaria, pero seriamente autolimitada a ser un ghetto de refugiados académicos (Vanee, 1983; Irvine, 1990).

Ante tales inhibiciones, quizá no sorprende que el reciente desarrollo del discurso no esencialista y más cultural acerca de la sexualidad no haya irrumpido en el centro de la antropología sino en su periferia, proveniente de otras disciplinas (especialmente de la historia), y de teorizaciones elaboradas por grupos marginales. La proliferación de trabajos estimulantes y desafiantes que en los últimos 15 años han seguido la llamada teoría de la construcción social, todavía no se ha manifestado por completo en las principales corrientes de la antropología.

La historia intelectual de la teoría de la construcción social es compleja y los ejemplos que aquí se ofrecen son una mera ilustración, sin pretender hacer una revisión completa. ${ }^{2}$ La teoría de la construcción social se ha basado en el desarrollo de varias disciplinas: dentro

${ }^{1}$ Esta resistencia puede tener efectos paradójicos, a juzgar por una experiencia personal. Mi propia solicitud para obtener un apoyo para terminar una convencional bibliografia comentada sobre las influencias bioculturales en la sexualidad fue rechazada argumentando que la investigadora "era muy joven para involucrarse en esta problemática" y que, siendo incapaz de leer japonés "no podría leer en el idioma original la nueva e importante bibliografia sobre los macacos japoneses". Lejos de desalentarme, estos comentarios motivaron más mi interés, pues parecía que las volubles reacciones de los antrópologos encerraban al menos tanto misterio como el propio material comparativo de mi investigación.

${ }^{2}$ Para los textos básicos, véase Katz, 1976 y 1983; Weeks, 1977; D'Emilio y Freedman, 1988; Altman et al., 1989; Duberman et al., 1989. 
de la sociología, en la sociología del interaccionismo social (social interactionism), en la teoría de la etiquetación (the labeling theory) y en la sociología de las conductas desviadas (deviance) (Gagnon y Simon, 1973; Plummer, 1982); en el campo de la historia, la historia social, los estudios laborales (labor studies), historia de las mujeres e historia marxista (Duggan, 1990); y en el campo de la antropología, en la antropología simbólica, los estudios comparativos de la sexualidad (cross-cultural works on sexuality) y los estudios de género -sólo se han nombrado las corrientes más significativas-. Además, las teorizaciones en muchas disciplinas son la respuesta a nuevos cuestionamientos elaborados por académicos y académicas feministas, gays y lesbianas preocupados por el género y la identidad.

\section{Sexualidad y género}

Activistas y académicas feministas emprendieron el proyecto de repensar el género, lo cual tuvo un impacto revolucionario sobre las nociones de lo considerado como natural. Los esfuerzos feministas se enfocaron en una revisión crítica de las teorías que utilizaban a la reproducción para relacionar el género con la sexualidad, explicando así la inevitabilidad y naturalidad de la subordinación de las mujeres (para la antropología, véase Reiter, 1975; Rosaldo y Lamphere, 1974; Lamphere, 1977; Rapp, 1979; Atkinson, 1982; Moore, 1988).

La reexaminación teórica abrió paso a una crítica general del determinismo biológico, en particular del conocimiento adquirido sobre la biología de las diferencias sexuales (Bleier, 1984; Fausto-Sterling, 1985; Sayers, 1982; Lowe y Hubbard, 1983; Hubbard $e$ t al., 1982; Tobach y Rosoff, 1978). Las evidencias comparativas, culturales e históricas mostraron la poca claridad de la noción, según la cual los roles de la mujer -que han tenido amplia variación- hubieran sido causados por la sexualidad y la reproducción humanas, en apariencia uniformes. A la luz de la diversidad de los roles de género de la sociedad humana, resultó improbable que éstos fueran inevitables o causados por la sexualidad. Pero la facilidad con que tales teorías se aceptaban sugiere ahora que la ciencia fue conducida y mediada por fuertes creencias acerca del género, que a su vez brindaron apoyo ideológico a las relaciones sociales en curso. Más aún, la creciente sensibilidad ante los aspectos ideológicos de la ciencia abrió el camino a un cuestionamiento de mavor alcance acerca de las conexiones históricas entre la 
dominación masculina, la ideología científica y el desarrollo de la ciencia y la biomedicina en Occidente (Harding, 1986; Schiebinger, 1989; Ehrenreich y English, 1979; Barker-Benfield, 1976; Haraway, 1989; Jordanova, 1989; Keller, 1984; Harding y Hintikka, 1983).

La práctica feminista de activismo en grupos de base también fomentó análisis que separaron la sexualidad del género. Las numerosas batallas para lograr el acceso de las mujeres al aborto y al control natal representaron una tentativa de separar la sexualidad de la reproducción y de los roles genéricos como esposas y madres. Las discusiones que elevaban la conciencia en los grupos clarificaron que un cuerpo que naturalmente parecía pertenecer a un género era de hecho un producto altamente mediado por la sociedad: el atractivo sexual y la feminidad eran alcanzados por medio de un persistente proceso de socialización donde se incluían estándares de belleza, maquillaje y lenguaje corporal. Finalmente, las discusiones entre diferentes generaciones de mujeres clarificaron cuán variable era su supuesta sexualidad natural, que se había movido, en nuestro siglo, desde el deber marital hasta el orgasmo múltiple, del erotismo vaginal al del clítoris y de la desapasionada era victoriana al entusiasmo femenino correspondiente a los cambios sociales. Al parecer, la sexualidad y el género ibanijuntos, pero en vías sujetas al cambio.

En 1975, el influyente ensayo de la antropóloga Gayle Rubin, "The Traffic in Women", produjo un apasionante argumento en contra de las explicaciones esencialistas, las cuales consideraban que la sexualidad y la reproducción habían causado, simple e inevitablemente, las diferencias de género (Rubin, 1975). En lugar de ello, Rubin exploró la estructura del "aparato social sistemático que toma a las mujeres como materia prima y forma mujeres domesticadas como productos" (Rubin, 1975: 158). La autora propuso el término "sistema sexo/género" para describir "el conjunto de arreglos por los cuales la sociedad transforma la sexualidad biológica en productos de la actividad humana, en los cuales se satisfacen estas necesidades sexuales" (Rubin, 1975: 159).

En 1984, Rubin sugiere una posterior desconstrucción del sistema sexo/género en dos dominios separados, en los cuales la sexualidad y el género serían reconocidos como sistemas diferenciados (Rubin, 1984). La mayoría de los análisis feministas previos consideraba a la sexualidad como una categoría totalmente derivada, cuya organización estaba determinada por la estructura de la inequidad de género. De acuerdo con la formulación de Rubin, la sexualidad y el género 
son fenómenos analíticamente diferentes, los cuales requieren andamiajes explicativos separados, aun cuando se hallen relacionados entre sí por circunstancias históricas específicas. Las teorias sobre la sexualidad no pueden explicar el género, y tomando el argumento en un nuevo nivel, las teorías sobre el género no pueden explicar la sexualidad.

Esta perspectiva sugiere un nuevo entramado: la sexualidad y el género son sistemas separados aunque entretejidos en muchos puntos. Aun cuando los miembros de una cultura experimenten tal entretejido como natural, orgánico y sin costuras, los puntos de conexión varían histórica y culturalmente. Para los investigadores de la sexualidad, la tarea no consiste sólo en estudiar los cambios de expresión de la conducta y las actitudes sexuales, sino en examinar las relaciones entre estos cambios y los movimientos más profundos en la organización del género y la sexualidad, dentro del contexto de relaciones sociales más amplias.

\section{Sexualidad e identidad}

Un segundo estímulo para el desarrollo de la teoría de la construcción social surgió a partir de los problemas que emergieron al examinar la homosexualidad masculina en Estados Unidos y Europa en el siglo xIx (Katz, 1976 y 1983; Weeks, 1977 y 1981). Es interesan te notar que una parte significativa de esta investigación pionera fue conducida por estudiosos independientes, no académicos, o por académicos disidentes que trabajaban generalmente sin recursos ni apoyo universitario, ya que en ese momento la historia de la sexualidad (particularmente la de los grupos marginales) dificilmente se consideraba un tema legítimo. Ahora que esta investigación recientemente ha alcanzado una módica aceptación académica, se ha convertido en lugar común entre los académicos apegados a lo institucional el dar créditos de estos desarrollos a Foucault y su Historia de la sexualidad (Foucault, 1978). Sin negar esta contribución, el hacer una genealogía tan singular oscurece un origen importante de la teoría de la construcción social y otorga créditos inmerecidos a las universidades y las disciplinas por un desarrollo que nunca apoyaron.

El primer intento de tratar con cuestiones de identidad sexual en una vía que hoy es reconocible como construcción social apareció en el ensayo de 1968 de Mary Mclntosh sobre el rol homosexual en Inglaterra 
(Mclntosh, 1968). Este artículo, que marca un camino a seguir, ofrece muchas intuiciones sugerentes acerca de la construcción histórica de la sexualidad en Inglaterra; sin embargo, sus observaciones se desvanecieron como piedrita en el estanque, hasta la mitad de la década de los años setenta, cuando nuevamente fueron consideradas por escritores preocupados por el feminismo y la liberación. Es por estos años que aparece una aproximación identificable ya como construccionista.

Los primeros trabajos de la historia gay y lésbica trataron de recuperar y revivir documentos, narraciones y biografias que estaban perdidos o eran ignorados, tanto por negligencia histórica como por los activos esfuerzos de archivistas, historiadores y gobiernos por suprimir tales materiales. Estos documentos y su contenido fueron concebidos primeramente como "lésbicos" o "gays" y la recuperación como una búsqueda de sus raíces históricas. Debe reconocerse que los investigadores que comenzaron esta empresa y compartían la ideología cultural implícita acerca de categorías sexuales fijas, después consideraron otras maneras de vislumbrar sus materiales y se plantearon interrogantes de mayor alcance.

Jeffrey Weeks (1977), historiador inglés de la sexualidad, fue el primero que articuló esta transición teórica. A partir del concepto de rol homosexual que desarrolla Mclntosh, Weeks distinguió entre conducta homosexual -a la cual consideró como universal- e identidad homosexual, a la cual visualizó como algo cultural e históricamente especifico del desarrollo relativamente reciente en la Gran Bretaña. Con un rico y provocador análisis de identidades y actitudes cambiantes, Weeks contextuó también la sexualidad, mostrando sus relaciones con la reorganización de la familia, del género y del grupo doméstico en el siglo xix británico.

El trabajo de Jonathan Katz también siguió este proceso. Su primer libro se ubica en la tradición de búsqueda de ancestros gay (Katz, 1976). Sin embargo, durante la elaboración de su segundo libro, Katz comenzó a considerar que los actos de sodomía consignados en documentos coloniales de Estados Unidos a partir del siglo xvi no eran equivalentes a la homosexualidad contemporánea (Katz, 1983). Parece que la sociedad colonial no concebía un tipo único de personas -un homosexual- comprometido con estos actos. Tampoco apareció alguna evidencia de una subcultura homosexual o de individuos cuyo sentido subjetivo de identidad fuera organizado con base en lo que nosotros entendemos como preferencia o identidad sexual. El segundo libro de Katz muestra una clara distancia del primero, 
pues los registros o relatos que documentan relaciones emotivas o sexuales entre personas del mismo sexo no son considerados como evidencias de identidad "gay" o "lésbica", sino como el punto de partida de una serie completa de interrogantes acerca del significado de tales actos para las personas que en ellos participaban, así como para la cultura y el momento en el cual vivieron.

Estos desarrollos intelectuales son también evidentes en los primeros trabajos sobre la formación de la identidad lésbica (Sahli, 1979; Rupp, 1980; Faderman, 1981; Rubin, 1979) y en los estudios que consideran cuestiones sobre la identidad y la conducta sexuales en culturas no occidentales, por ejemplo, el trabajo de Gilbert Herdt en Nueva Guinea (Herdt, 1981, 1984 y 1987). Tras un creciente número de trabajos (Weeks, 1977; Plummer, 1981; D'Emilio, 1983; Bray, 1982; Newton, 1984; Davis y Kennedy, 1986; Vicinus, 1989; Gerard y Hekma, 1988) siguió una impresionante disposición a la imaginación: las categorías "homosexual" y "lesbiana", ¿habían existido siempre?, y si no, ¿cuáles fueron sus orígenes y las condiciones para su desarrollo? Si los actos físicos idénticos tienen diferentes significados subjetivos, ¿cómo se construyen los significados sexuales? Si las subculturas sexuales se interiorizan en el ser, ¿qué es lo que guía su formación? Y aunque estas cuestiones fueron formuladas inicialmente en términos de la historia y la identidad homosexuales, es claro que eran igualmente aplicables a la historia e identidad heterosexuales, implicación que ahora está, precisamente, siendo explorada (Peiss, 1983 y 1986; Stanseli, 1986; Trimberger, 1983; Katz, 1990).

La sexualidad como un campo en disputa

Los trabajos sucesivos sobre la historia de la construcción de la sexualidad en la sociedad estatal moderna muestran que la sexualidad es un campo sujeto a la activa disputa política y simbólica, sobre el cual distintos grupos luchan por instrumentar programas sexuales y cambiar las disposiciones e ideologías sexuales. En los siglos xIx y xx, el crecimiento del interés estatal para regular la sexualidad (y el relativo declive del control religioso) convirtieron al parlamento y a los programas públicos de gobierno en campos particularmente atractivos para las batallas políticas e intelectuales alrededor de la sexualidad. Los movimientos masivos en torno de las enfermedades venéreas, la prostitución, la masturbación, la pureza social, y la existencia de nor- 
mas distintas para cada sexo (doble estándar) han formado organizaciones políticas, han hecho cabildeos parlamentarios, han convocado a demostraciones masivas e incidido en la cultura por medio de complejos símbolos, representaciones y retórica (Weeks, 1981; Peiss y Simmons, 1989; Walkowitz, 1980; Bristow, 1977; Pivar, 1972; Brandt, 1985; Kendrick, 1987; Gordon, 1974). Dado que la participación del Estado fue crecientemente formulada en un lenguaje sobre la salud, los médicos y científicos se convirtieron en participantes importantes en los desarrollos de los nuevos discursos regulatorios. Su activa participación en la formulación de estos discursos fue también una vía para legitimar sus nuevas especialidades profesionales.

Aunque los grupos socialmente poderosos ejercieron más poder discursivo, no fueron los únicos participantes en las batallas sexuales. Grupos minoritarios progresistas, reformistas, sufragistas y radicales sexuales también presentaron propuestas de cambio e introdujeron nuevas vías para pensar y organizar la sexualidad. Las subculturas sexuales que crecieron en áreas urbanas fueron un campo especialmente fértil para estos experimentos. Los estudios construccionistas muestran cómo los intentos de construir espacios públicos parcialmente protegidos, en los cuales se elaboren y expresen nuevas formas, conductas y sensibilidades sexuales, han sido también parte de una batalla política de gran escala para definir la sexualidad. Las subculturas no sólo hacen crecer nuevas vías para organizar la identidad y la conducta, sino también nuevos caminos de resistencia simbólica y ajuste del orden dominante, algunos de los cuales llegan a tener un profundo impacto más allá de los pequeños grupos en los cuales se produjeron. En este sentido, el trabajo de construcción social ha sido valioso por explorar la mediación humana y la creatividad en la sexualidad, alejándose de modelos unidimensionales de cambio social para describir complejas y dinámicas relaciones entre el Estado, los expertos profesionales y las subculturas sexuales. Este intento de situar la sexualidad en un marco histórico ha producido un innovador conjunto de trabajos, al que han contribuido historiadores, antropólogos, sociólogos y otros estudiosos en un diálogo interdisciplinario poco frecuente.

El desarrollo de los modelos de construcción social, 1975-1990

La creciente popularidad del término "construcción social" oscurece el hecho de que los escritores construccionistas lo hayan usado en 
sentidos diferentes. Es verdad que todos rechazan las definiciones transhistóricas y transculturales de sexualidad y en su lugar sugieren que la sexualidad está mediada por factores históricos y culturales. Pero una lectura cuidadosa de los textos construccionistas muestra que los construccionistas sociales difieren en sus visiones de lo que puede ser construido, variaciones que incluyen actos sexuales, identidades sexuales, comunidades sexuales, la dirección del interés erótico (elección de objeto), y el deseo sexual mismo. A pesar de estas diferencias, todos comparten la urgencia de cuestionar los términos y el campo de estudio.

Como mínimo, todas las aproximaciones de la construcción social adoptan el punto de vista de que actos sexuales fisiológicamente idénticos pueden tener significación social y significado subjetivo variable, dependiendo de cómo sean definidos y entendidos en periodos históricos y culturas diferentes. Dado que un acto sexual no lleva consigo un significado social universal, la relación entre actos y significados sexuales no es fija, y existe el gran peligro de que al estudiarlos, el observador proyecte sus propios tiempo y espacio. Las culturas proporcionan una amplia variedad de categorías, esquemas y etiquetas para conformar las experiencias sexuales y afectivas. Estas construcciones influyen no sólo en la conclucta y la subjetidad individuales, sino también organizan y dan significado a la experiencia sexual colectiva por medio de, por ejemplo, impactar identidades, definiciones, ideologías y regulaciones sexuales. La relación entre actos e identidades sexuales en comunidades sexualmente organizadas es igualmente variable y compleja. Así, estas distinciones entre actos sexuales, identidades y comunidades es utilizada ampliamente por los escritores construccionistas.

$\mathrm{Al}$ avanzar un paso más, la teoría de la construcción social establece que la dirección misma del deseo erótico, por ejemplo la elección del objeto (heterosexualidad, homosexualidad y bisexualidad, como la sexología contemporánea lo conceptuaría), no es inherente o intrínseco al individuo, sino que es construido desde las más polimorfas posibilidades. No todos los construccionistas dan este paso, y para quienes no lo hacen, la dirección del deseo y del interés erótico puede ser pensada como algo fijo, aunque la forma conductual que este interés tome estará construida por los andamiajes culturales prevalecientes, lo mismo que la experiencia subjetiva de los individuos y la significación social que los otros le atribuyan. 
La forma más radical de la teoría construccionista ${ }^{3}$ está dispuesta a considerar la idea de que no hay un "impulso" sexual esencial e indiferenciado, un "impulso sexual" (sexual drive) o "lujuria" que residan en el cuerpo, debido a una sensación o funcionamiento fisiológico. El deseo sexual, entonces, es en sí mismo construido por la cultura y la historia desde las energías y capacidades del cuerpo. En este caso, una cuestión construccionista importante se refiere al origen de estos impulsos, desde que se asume que ellos no son intrínsecos, y quizá ni necesarios. Esta posición, por supuesto, contrasta radicalmente con una teoría construccionista más intermedia o mediadora, la cual implícitamente acepta un deseo inherente que luego será construido en términos de actos, identidad, comunidad y elección de objeto. El contraste entre las posiciones mediadora y radical evidencia que los construccionistas bien pueden discutir entre sí, como también pueden hacerlo con los trabajos de las tradiciones esencialistas y de influencia cultural. Sin embargo, la bibliografía de la construcción social, cuya aparición inicial fue a mediados de los años setenta, muestra un desarrollo gradual en la habilidad de imaginar que la sexualidad es construida.

\section{Modelos de influencia cultural sobre la sexualidad, 1920-1990}

En contraste, las aproximaciones convencionales a la antropología de la sexualidad, desde 1920 hasta 1990, mantienen una notable persistencia. Al igual que la sexualidad se mantiene como un constructo no examinado, los fundamentos teóricos permanecen sin examinar, sin ser nombrados e, implícitamente, como si ellos fueran tan inevitables y naturales que podría haber poca disputa o elección en una aproximación estándar, casi genérica. Por esta razón quiero sugerir el nombre de "modelo de influencia cultural" para llamar la atención sobre los rasgos distintivos y promover mayor reconocimiento de este paradigma. En este modelo la sexualidad es vista como la materia prima, como una especie de plastilina universal sobre la cual trabaja la cultu-

${ }^{3}$ No se sugiere aquí que las formas más radicales de la teoría de la construcción social sean necesariamente las mejores, aunque el ejercicio de desconstrucción total de una de las categorías más esenciales, la sexualidad, suele tener un efecto electrificante y energizador en el pensamiento. El hecho de que este grado de desconstrucción pueda mantenerse plausiblemente, es una cuestión distinta. 
ra, como una categoría natural que permanece cerrada a la investigación y al análisis.

Por otro lado, el modelo de influencia cultural enfatiza el papel de la cultura y del aprendizaje para conformar las actitudes y la conducta sexual. En este sentido, rechaza formas obvias de esencialismo y universalismo. La variación ha sido un descubrimiento clave en muchos de sus estudios: encuestas comparativas (Ford y Beach, 1951; Minturn, Grosse y Haider, 1969; Broude y Greene, 1976; Gray, 1980; Frayser, 1985), relatos etnográficos de sociedades cuyas costumbres sexuales son claramente distintas de las comunes para el lector europeo o estadunidense (Mead, 1923; Malinowski, 1941; Schapera, 1941; Goodenough, 1949; Berndt y Berndt, 1951; Levine, 1959; Howard y Howard, 1964; Davenport, 1965; Suggs, 1966; Lessa, 1966; Marshall y Suggs, 1972; Heider, 1976; Marshall, 1976), así como en recuentos teóricos generales (Goldenweiser, 1929; Bateson, 1947; Murdock, 1949; Honigman, 1954; Gerbhard, 1976). La cultura es percibida como un estimulante o bien como inhibidora de actitudes, relaciones y actos sexuales genéricos. El contacto genital/oral, por ejemplo, puede ser parte de la expresión heterosexual normal en un grupo, o ser tabú en otro; el hombre homosexual puede ser severamente castigado en una tribu aunque tolerado en otra. El trabajo antropológico en este periodo se caracterizó por su interés permanente en la variabilidad.

Por otro lado, aunque se piense que la cultura configura las costumbres y la expresión sexual, se asume -frecuentemente de modo explícito- que, fundamentalmente, la sexualidad está determinada universal y biológicamente; en la bibliografia sobre el tema aparece como "impulso" o "impulso sexual" (sexual drive) ${ }^{4}$ Aunque es suceptible de ser configurado, este instinto -o pulsión- se concibe como algo poderoso, que se mueve hacia su expresión después de su despertar en la pubertad, que algunas veces excede las regulaciones sociales y que toma formas distintivas en hombres y mujeres.

El corazón de la sexualidad es la reproducción. Aunque la mayoría de los relatos antropológicos no busca restringirse al análisis de la conducta reproductiva, la sexualidad reproductiva (glosada como unión heterosexual) suele aparecer como la carne y las papas del menú sexual, mientras que otras formas, tanto homosexuales como hetero-

${ }^{4}$ El trabajo de Heider (1976) constituye una excepción, pues su conceptuación considera niveles variables de energía sexual. 
sexuales, son presentadas como los aperitivos, las ensaladas y el postre (estas metáforas no son desconocidas en las narraciones antropológicas). Estos estudios y relatos etnográficos siguen casi siempre el mismo orden de exposición, que primero trata sobre el "sexo real" y luego se dirige a las "variaciones". Algunos relatos, que supuestamente tratan de la sexualidad, son notoriamente sucintos al detallar la conducta no reproductiva; el artículo de Margaret Mead acerca de las determinantes culturales de la conducta sexual (en un volumen con el maravilloso título de Sex and Intermal Secretions de 1961) hace un vertiginoso recorrido que incluye embarazo, menstruación, menopausia y lactancia, pero muy poco sobre erotismo y sexualidad no reproductiva. De manera similar, un libro más reciente, con el extenso título de Varieties of Sexual Experience (1985) dedica virtualmente toda la obra, excepto algunas páginas, al matrimonio, la reproducción y la organización familiar (Frayser, 1985).

Dentro del modelo de influencia cultural, el término "sexualidad" cubre un amplio rango de temáticas. Su significado frecuentemente se da por sabido, como un entendido implícito, que comparten el autor y el lector. Al buscar su uso en varios artículos y libros se muestra que la sexualidad incluye muchos conceptos muy diferentes: relaciones sexuales, orgasmo,juegos y caricias previas al coito (foreplay), humor, historias y fantasías eróticas, diferencias sexuales y organización de la masculinidad y feminidad, relaciones de género (regularmente llamadas "roles sexuales" en los primeros trabajos).

En este modelo la sexualidad no sólo está relacionada con el género, sino que se mezcla y fácilmente se confunde con él. Sexualidad, arreglos de género, masculinidad y feminidad se asumen como conectados, e incluso se consideran intercambiables. Esta suposición, sin embargo, no ilumina sus conexiones históricas y culturales específicas, sino que las oscurece. La confusión surge de nuestras propias creencias folklóricas, según las cuales, en primer lugar, el sexo causa al género, esto es, las diferencias reproductivas entre el hombre y la mujer, así como el proceso de reproducción (equiparado con "sexualidad") hacen crecer las diferencias de género; en segundo lugar, el género causa al sexo, es decir, las mujeres como un grupo genéricamente marcado constituyen el lugar de la motivación, el deseo sexual y la sexualidad. La reproducción y su organización se convierten en el móvil inicial de toda diferenciación entre macho y hembra, y del florecimiento del sistema de género. Así, el género y la sexualidad se tejenijuntos, sin costura alguna que los diferencie. 
Finalmente, el modelo de influencia cultural asume que los actos sexuales conllevan una significación estable y universal en términos de identidad y significado subjetivo. Comúnmente, en la bibliografía especializada se considera al contacto sexual entre géneros opuestos como "heterosexualidad" y al contacto con el mismo género como "homosexualidad", como si el mismo fenómeno fuese observable en todas las sociedades en las que estos actos ocurren. Analizados a posteriori, estos presupuestos son curiosamente etnocéntricos, dado que el significado atribuido a estas conductas sexuales proviene del observante y de la compleja sociedad industrial del siglo $\mathrm{xx}$. Las encuestas comparativas permitieron elaborar mapas que muestran la distribución geográfica de los contactos sexuales entre el mismo género o géneros opuestos, o la frecuencia de los contactos sexuales previos al matrimonio. Sin embargo, cuando los investigadores utilizan estos trabajos para comunicar la presencia o ausencia de "homosexualidad" o "permisividad sexual", están haciendo una identificación espuria entre el acto o conducta y el significado o identidad sexual, traducción que el desarrollo teórico posterior ha rechazado.

En suma, el modelo de influencia cultural reconoce variaciones en la existencia de conductas sexuales y actitudes culturales que fomentan o restringen la conducta, pero no en el significado mismo de la conducta. Adicionalmente, los estudios antropológicos dentro de este andamiaje conceptual aceptan sin cuestionamiento la existencia de categorías universales como homosexual y heterosexual, sexualidad masculina y femenina, e impulso sexual.

A pesar de las numerosas deficiencias anotadas, es necesario reconocer la fuerza de esta aproximación, particularmente en sus contextos intelectual, histórico y político. El compromiso de la antropología con la comparación cultural hizo que esta disciplina fuera la más relativista de las ciencias sociales en relación con el estudio de la sexualidad. Sus descubrimientos sobre la variación pusieron en entredicho las nociones prevalecientes acerca de la inevitabilidad o naturalidad de las normas y la conducta sexual en Estados Unidos y Europa, así como la conexión entre la regulación sexual y la estabilidad social o familiar. La variabilidad reportada sugirió que la sexualidad humana era maleable y capaz de asumir formas diversas. Los trabajos en la tradición de la influencia cultural abarataron teorías más mecanicistas de la conducta sexual, aún comunes en medicina y psiquiatría, que sugieren que la sexualidad es, en gran medida, una función fisiológica o un impulso instintivo. La idea de la variación sexual comenzó a 
desarrollar espacios sociales e intelectuales en los cuales era posible considerar a la sexualidad como algo más que una simple función de la biología.

Aunque el trabajo realizado por el modelo de influencia cultural contribuyó al desarrollo de la teoría de la construcción social, hay entre ambos una clara ruptura en muchos aspectos. Las diferencias no han sido reconocidas por numerosos antropólogos que siguen trabajando dentro de la tradición de la influencia cultural. Más aún, hay muchos malentendidos al considerar que los nuevos desarrollos son teóricamente compatibles, o que son la continuación de estudios anteriores. Algunos han asimilado en sus trabajos términos o frases (como "construcción social" o "construcción cultural"), a pesar de que su andamiaje analítico continúa sin cuestionar muchos elementos esencialistas. ${ }^{5}$ Aunque el modelo de influencia cultural reconoce la variación cultural, no es lo mismo que la teoría de la construcción social. Por tanto, el modelo de influencia cultural ya no se mantiene como el único paradigma antropológico, aunque siga dominando los trabajos contemporáneos (Frayser, 1985; Mascia-Lees, 1989).

Parece que el desarrollo de la antropología en este siglo -un movimiento general que ha pasado de las tendencias biologizantes a las perspectivas desnaturalizantes y antiesencialistas-favorecería la aplicación de la teoría de la construcción social al estudio de la sexualidad. No obstante, si la antropología ha desafiado en muchos terrenos al estatus de lo natural y universal, en gran medida la sexualidad ha quedado excluida del esfuerzo para sugerir que las acciones humanas han estado sujetas a fuerzas históricas y culturales y, por tanto, al cambio.

${ }^{5}$ Una manera diferente de asimilar esta controversia sostiene que el debate entre construccionistas y esencialistas es una nueva variante de la polémica " ¿naturaleza o educación?". Éste es un profundo malentendido de la teoría de la construcción social.

En los debates ¿naturaleza o educación?, los investigadores proponen mecanismos alternativos, o biológicos o culturales, para explicar el fenómeno que observan. En la actualidad, la mayoría de los observadores que aceptan que la conducta humana es producida por una compleja interacción de factores biológicos y culturales difieren en el peso relativo que asignan a cada uno.

Aunque parezca apropiado encontrar alguna semejanza entre los esencialistas y el campo de la naturaleza, equiparar la construcción social al campo de la educación es un error. La teoría de la construcción social no arguye simplemente a favor de la causalidad cultural. Además, y más importante, nos estimula a deconstruir y examinar la conducta o el proceso por el cual ambos campos, el natural y el educativo han reificado y quieren "explicar". La construcción social sugiere que el objeto de estudio merece al menos tanta atención analítica como el sospechoso mecanismo causal: 
Una aproximación a la construcción social de la sexualidad examinaría el rango de conducta, ideología y significado subjetivo entre y dentro de los grupos humanos, y examinaría al cuerpo, a sus funciones y sensaciones como potencialidades (y como límites) que están incorporados y mediados por la cultura. La fisiología del orgasmo y de la erección del pene explica el esquema sexual de una cultura tanto como el rango auditivo del oído explica la música. La biología y las funciones fisiológicas son determinantes sólo en los límites más extremos, y es allí donde establecen el límite de lo que es fisiológicamente posible. La cuestión más interesante para la investigación antropológica sobre la sexualidad es situar geográficamente aquello que se considera como culturalmente posible, y esto constituye un campo mucho más extenso. De igual manera, la adaptación ecológica y las demandas reproductivas explican sólo una pequeña parte de la organización sexual, dado que ajustar la fecundidad para reemplazar y aun para hacer crecer una población es relativamente fácil de lograr para la mayoria de los grupos. Lo que es más importante, la sexualidad no es coextensiva ni equivalente a la reproducción: la sexualidad reproductiva constituye una pequeña parte de un universo sexual mayor.

Además, la aproximación a la construcción social de la sexualidad debe problematizar y cuestionar también las creencias científicas y folklóricas europeas y estadunidenses acerca de la sexualidad, en lugar de proyectarlas sobre otros grupos de una manera etnocéntrica, inaceptable en cualquier otro campo de estudio. Así, afirmaciones sobre la fuerza compulsiva universal del impulso sexual, la importancia de la sexualidad para la vida humana, el estatus privado y universal de la conducta sexual, o la quintaesencia de la naturaleza reproductiva requieren ser presentadas como hipótesis, no como supuestos a priori. La antropología parece estar especialmente bien situada para problematizar la supuesta naturalidad de la mayoría de estas categorías; sin embargo, la sexualidad ha sido el último dominio (aun posterior al género) cuyo estatus natural y biologizado ha sido cuestionado. Para muchos de nosotros, el esencialismo fue la primera vía para pensar en la sexualidad, y éste continúa siendo hegemónico.

La teoría de la construcción social ofrece una perspectiva radicalmente diferente para el estudio de la sexualidad, animando nuevas y fructíferas investigaciones. Su influencia ha ido creciendo en la antropologia (Newton, 1979; Caplan, 1987; Davis y Kennedy, 1989; Whitehead, 1981; Blackwood, 1986; Fry, 1985; Carrier, 1985; Vanee, 1990; Parker, 1991), aunque el modelo de influencia cultural aún domina 
(Frayser, 1985 y 1989; Gregor, 1985; Cohen y Mascia-Lees, 1989; Mascia-Lees, Tierson y Relethford, 1989; Perper, 1989). Se podría predecir una gradual intensificación de la competencia entre paradigmas, quizá incluso un cambio paradigmático. Sin embargo, la aparición del sida ha alterado esta dinámica.

Sida e investigación sobre sexualidad

La gran preocupación por el sida ha incrementado dramáticamente el interés en dirigir y financiar la investigación sobre sexualidad. Al principio de la epidemia, los epidemiólogos comenzaron a elaborar -siguiendo sus rutinas- conjuntos de preguntas relativas a la frecuencia y naturaleza de la conducta sexual. Sus problemas de medición y conceptuación, así como sus infructuosos resultados sobre esta base de datos evidenciaron la negligencia de la investigación científica sobre lo sexual. Además, el hecho de que estudios en gran escala sobre los hábitos sexuales estadunidenses no se hubieran realizado desde los volúmenes de Kinsey (Kinsey, Pomeroy y Martin, 1948; Kinsey, Pomeroy, Martin y Gebhard, 1953) dio lugar a un gran apuro, mostrando nuestra incapacidad para responder siquiera a las cuestiones más elementales. Cuando científicos y políticos reconocieron la necesidad de esta información, hicieron fuertes recomendaciones para incrementar drásticamente los fondos y los esfuerzos de investigación en los países afectados (Turner, Miller y Moses, 1989; Booth, 1989a y 1989b). Aunque en muchos sentidos esto es un paso positivo y necesario, la precipitación para financiar investigaciones incrementa la posibilidad de revivir y robustecer modelos inadecuados, sean esencialistas o de influencia cultural sobre la sexualidad.

El sida favorece el resurgimiento de las aproximaciones biomédicas a la sexualidad mediante la repetida asociación de sexualidad y enfermedad. La medicalización de la sexualidad es intensificada cuando el público voltea hacia las autoridades médicas en busca de información y consejo. Además, los investigadores biomédicos de las escuelas de medicina y salud pública están conduciendo una parte significativa de la investigación sobre sexualidad, relativa al sida. ${ }^{6}$ Esto marca un cambio

${ }^{6}$ Esto no significa que no haya investigaciones conducidas por científicos sociales fuera de las instituciones médicas, ni que los científicos sociales no estén también contribuyendo a los estudios realizados por las escuelas médicas, aunque generalmente su 
de la tendencia general desarrollada después de la segunda guerra, en que la investigación sobre sexualidad se movió cada vez más fuera de las arenas médicas. Ahora, el interés médico sobre la sexualidad se está expandiendo a nuevas áreas, más allá de las especialidades en que había estado tradicionalmente confinado: enfermedades de transmisión sexual, ginecología y obstetricia, y psiquiatría.

Este desarrollo conlleva varios peligros. Las aproximaciones biomédicas a la sexualidad consideran frecuentemente a la sexualidad como una derivación de la fisiología y de un supuesto funcionamiento universal del cuerpo. Los modelos biomédicos tienden a ser más irreflexivos acerca de la influencia de la práctica científica y médica en construir categorías tales como "el cuerpo" y "la salud". Las aproximaciones de la construcción social son virtualmente desconocidas, y la noción de que la sexualidad varía con la cultura y la historia es expresada, en el mejor de los casos, mediante los primitivos modelos de influencia cultural. Hay un limitado reconocimien to de que la sexualidad tiene una historia y de que sus definiciones y significados cambian en el tiempo y dentro de las poblaciones. La confianza de la investigación biomédica en instrumentos tales como las encuestas y en datos fácilmente cuantificables incrementa la tendencia a contar actos en vez de explorar significados. Por ejemplo, dichos estudios han equiparado frecuentemente actos sexuales con identidades sexuales, y han hablado de "gays" y "heterosexuales" como categorías no problemáticas. Además, el elevado estatus de los practicantes de la medicina en el siglo $\mathrm{xx}$ y su reclutamiento entre clases, géneros y grupos raciales privilegiados, ha conducido, históricamente, a su cercana alianza con ideologías dominantes, incluyendo la sexual. Si tal patrón persiste, es improbable que este grupo sea consciente de las sensibilidades y subculturas sexuales marginales, y no sea sensible a ellas.

Al organizar la investigación sexual dentro de un modelo biomédico y con la perspectiva de una enfermedad, también existe la ame-

rol sea de menor importancia. Sin embargo, el simple número de poblaciones investigadas con una orientación biomédica, unido a sus muestreos en gran escala y sus grandes presupuestos, amenazan con eclipsar y desplazar investigaciones sobre sexualidad conducidas por investigadores con una orientación menos bionédica. Además, los médicos con grado de doctorado son considerados con más a tutoridad que los científicos sociales para hablar sobre el cuerpo. Con todo esto, las perspectivas esencialistas que estudian la sexualidad, en relación con el sida, como un astmio corporal, tendrán un crecimiento que automáticamente incrementará la legitimidad de los textos y voceros médicos. 
naza de patologizar de nuevo a la sexualidad. Esto promete el regreso de la sexualidad a la posición que ocupaba a fines del siglo xix y principios del xx, cuando su discusión pública estaba en gran medida motivada y circunscrita al discurso de la enfermedad venérea, la prostitución y la masturbación. Estas discusiones públicas organizadas por expertos médicos, dirigidas ostensiblemente a tratar sobre salud y enfermedad, eran, implícitamente, discusiones sobre moralidad, género y orden social. El peligro se incrementa por el respeto concedido a la medicina y la ciencia y por la creencia pública de que la ciencia no contiene valores. La extensión de un discurso supuestamente objetivo y libre de valores acerca de la sexualidad, organizado bajo el disfraz de la salud, abre la puerta a un vasto crecimiento de la intervención gubernamental y profesional.

El énfasis puesto sobre los gays y su conducta sexual en las primeras etapas de la epidemia constituyó un claro distanciamiento de la situación previa, en la que los grupos sexuales subordinados eran más bien ignorados. Esta nueva atención, sin embargo, subraya la "otredad" de estos grupos, de una manera que recuerda los modelos patológicos del siglo pasado sobre homosexualidad (Gever, 1989), remarcando la naturalidad de su identidad y reforzando la abierta dicotomía entre homosexualidad y heterosexualidad. Esta otredad se va extendiendo a grupos adicionales estigmatizados como de alto riesgo de contraer el sida, tales como los usuarios de drogas intravenosas y sus parajas, o las mujeres de minorías de los barrios pobres del centro de las ciudades, trazándose así estereotipos que tienen resonancias históricas y culturales (Gilman, 1988).

El peligro planteado por el incremento de los fondos dedicados a investigar la sexualidad, en conexión con el sida, no se restringe a la biomedicina. Dentro de la antropología, es improbable que los modelos esencialistas reaparezcan; sin embargo, el campo puede experimentar el impacto del crecimiento de los enfoques biomédicos sobre la sexualidad, mediante los trabajos interdisciplinarios llevados a cabo por los establecimientos médicos. De mayor importancia es el hecho de que el incremento de los fondos y los urgentes llamados a la investigación, probablemente fortalezcan los modelos de influencia cultural en la sexualidad, en la medida en que un mayor número de antropólogos sean atraídos a investigar sobre el sida (Feldman y Johnson, 1986; Gorman, 1986; Bateson y Goldsby, 1988; Bolton, 1989; Marshall y Bennet, 1990).

La mayoría de los reclutados serán, probablemente, antropólogos médicos o especialistas en las áreas geográficas afectadas, sin en- 
trenamien to especializado en sexualidad. Como antropólogos, seguramente podrán brindar expectativas sobre la diversidad humana, sensibilidad hacia el etnocentrismo y respeto por el papel de la cultura en la conformación de las conductas, incluida la sexualidad. Pero éste es precisamente el problema, que estas perspectivas reinventarán el modelo de la influencia cultural, como una aproximación antropológica de sentido común hacia la sexualidad. Los antropólogos que son nuevos en el estudio de la sexualidad pueden fácilmente pensar que al admitir la variación cultural, su aproximación (basada en la influencia cultural) será idéntica a la teoría de la construcción social. Sus comparaciones con el trabajo hecho desde enfoques más biomédicos y biologizantes, particularmente en culturas no occidentales, hará que los modelos de influencia cultural les parezcan avanzados e incluso, un motivo para estar de plácemes.

En todos los campos de estudio, el reconocimiento tardío de las grandes lagunas existentes en el conocimiento de la conducta sexual puede enfatizar la importancia de los datos sobre el comportamiento, los cuales parecen ser más fácilmente medibles que la fantasía, la identidad o los significados subjetivos. Cuantificar la conducta facilita la medición y la fijación en esquemas metodológicos propios de la ciencia social positivista. En medio de una epidemia, los investigadores están presionados a obtener resultados rápidos y rechazan el tiempo, la paciencia y la tolerancia hacia la incertidumbre que las técnicas etnográficas y reconstructivas parecen requerir.

A pesar de estas tendencias, mismas que refuerzan las aproximaciones biologizantes y las de influencia cultural, el cuadro permanece complejo y contradictorio. Las investigaciones inspiradas por el sida en el mundo sexual real de los pueblos ya han revelado la existencia de discrepancias entre distintas ideologías sobre la sexualidad y la experiencia de vida. Las contradicciones se incrementan exponencialmente al estudiar otros contextos culturales. Las ambigüedades existen en muchas áreas, pero son particularmente notables al considerar los sistemas clasificatorios, la identidad, la congruencia entre conducta y autodefinición, el significado de los actos sexuales y la estabilidad de la preferencia sexual. Tales inconsistencias señalan la utilidad de la teoría de la construcción social y han estimulado nuevos trabajos en antropología (Parker, 1987; Murray y Payne, 1989; Carrier, 1989; Singer et al., 1990; Kane, 1990; Asencio, 1990; Hawkeswood, 1990). Tal como sucedió con los primeros estudios de la historia gay, los investigadores del sida y la sexualidad pue- 
den confrontar las limitaciones de sus modelos, generando un trabajo imaginativo y provocador.

Más aún, todo el fenómeno de "sexo seguro" ha enfatizado los aspectos culturalmente maleables de la conducta sexual. La campaña de sexo seguro montada por la comunidad gay, seguramente una de las campañas de salud pública más dramáticas y efectivas que se recuerden, clarificó que los actos sexuales sólo pueden ser entendidos dentro de un contexto cultural y subcultural y que una atención cuidadosa al significado y simbolismo permite la posibilidad de cambio, aun entre adultos (Patton, 1985; Altman, 1986; Crimp, 1989; Watney, 1987). El liderazgo autoconsciente y la participación de los hombres gay, opuestos a los expertos biomédicos, muestran el empeño de individuos activamente participativos, creando y cambiando significados culturales y eróticos, particularmente cuando tienen interés en hacerlo. La campaña de sexo seguro revela agentes sexuales activos, conscientes de su universo simbólico, y hábiles para manipularlo y recrearlo, en vez de receptores pasivos de una aculturación sexual estática.

Las movilizaciones política y simbólica alrededor de las dimensiones y significados sexuales del sida, como parte de muchos constitutivos diferentes, también desmienten la noción de que la sexualidad y su significado sean una simple derivación del cuerpo, inconmutable o fácilmente legible. El hecho de que varios grupos ofrezcan sus interpretaciones del sida y su significado sexual es una enseñanza sobre la naturaleza del cuerpo (Patton, 1985; Altman, 1986; Watney, 1987 y 1989; Grover, 1989; Treichler, 1989; Gilman, 1989; Treichler, 1988; Juhasz, 1990; Williamson, 1989). La multitud de lecturas en competencia y la ferocidad de la batalla sobre cuál de las interpretaciones prevalecerá, sugiere que el significado sexual es un campo -incluso político- que se encuentra en constante disputa. El hecho de que los sectores dominantes, particularmente el Estado, la religión y los grupos profesionales ejerzan una influencia desproporcionada sobre el discurso sexual no significa que sus visiones sean hegemónicas ni que otros grupos no las desafien. Tampoco significa que los grupos marginales sólo respondan reactivamente, sin crear sus propias subculturas y mundos de significado.

En la creación de nuevos discursos sobre la sexualidad es crucial que adquiramos conciencia de cómo estos discursos son creados y de nuestro propio papel en su creación. Los antropólogos tienen mucho que contribuir a la investigación de la sexualidad. La nueva situación producida por el sida en la investigación sexual está llena de posibili- 
dades: desarrollar la teoría de la construcción social sobre los nuevos desafios, o bien, regresar a los modelos esencialistas y de influencia cultural. Lo que está en juego no es poco para el estudio de la sexualidad, para la introducción de programas de educación y prevención del sida, para las políticas sexuales o para las vidas humanas. En este momento en que la an tropología "redescubre" al sexo, necesitamos considerar dos cuestiones: ¿quién será el encargado de observar? y, lo que es más importante, ¿qué es lo que seremos capaces de observar? Necesitamos ser explícitos acerca de nuestros modelos teóricos, cuidadosos de su historia y conscientes de nuestra práctica.

\section{Bibliografía}

Altman, D. (1986), ADs in the Mind of American: The Social, Political and Psychological Impact of a New Epidemic, Nueva York, Anchor Press/Doubleday.

, C. Vance, M. Vicinus y J. Weeks (eds.) (1989), Homosexuality, Which Homosexuality?, Amsterdam, An Dekker/Schorer.

Asencio, M. (1990), "Puerto Rican Adolescents Play by the Rules", trabajo presentado en la Convención Anual de la American Anthropological Asociation.

Atkinson, J. M. (1982), "Anthropology: A Review Essay", Signs, vol. 8.

Barker-Benfield, G. J. (1976), The Horrors of the Half-Known Life, Nueva York, Harper and Row.

Bateson, G. (1947), "Sex and Culture", Annals of the New York Academy of Science, vol. 47.

Bateson, M. C. y R. Goldsby (1988), Thinking ams: The Social Response to the Biological Threat, Reading, Mass., Addison-Wesley.

Berndt, R. M. y C. Berndt (1951), Sexual Behavior in Western Amhem Land, Nueva York, Viking Fund.

Blackwood, E. (ed.) (1986), Anthropology and Homosexuality, Nueva York, The Haworth Press.

Bleier, R. (1984), Sizence and Gender: A Critique of Biology and Its Theories on Women, Nueva York, Pergamon Press.

Bolton, R. (1989), "The Aibs Pandemic: A Global Emergency", Medical Anthro. pology, núm. 10 (número especial).

Booth, W. (1989a), "Asking America about its Sex Life", Science, vol. 242. (1989b), "Who Seeks Global Data on Sexual Practices", Science, vol. 244.

Brant, A. M. (1985), No Magic Bullet: A Social History of Venereal Disease in United States since 1880, Nueva York, Oxford University Press.

Bray, A. (1982), Homosexuality in Renaissance England, Londres, Gay's Men Press. 
Bristow, E. J. (1977), Vice and Vigilance: Purity Movements in Britain since 1700, Nueva Jersey, Rowman and Littlefield.

Broude, G. J. y S. J. Greene (1976), "Cross-Cultural Codes in Twenty Sexual Attitudes and Practices", Ethnology, vol. 15.

Caplan, P. (ed.) (1987), The Cultural Construction of Sexuality, Londres, Tavistock.

Carrier, J. M. (1985), "Mexican Male Bisexuality", Journal of Homosexuality, vol. 11.

(1989), "Sexual Behavior and the Spread of Ains in Mexico", Medical Anthropology Quarterly, vol. 10.

Cohen, C. B. y F. E. Mascia-Lees (1989), "Lasers in the Jungle: Reconfiguring Questions of Human and non-Human Primate Sexuality", Medical Anthropology, vol. 11.

Crimp, D. (ed.) (1989), ADs: Cultural Analysis, Cultural Activism, Boston, MIT Press.

Davenport, W. (1965), "Sexual Patterns and their Regulation in a Society of the South West Pacific", en F. Beach (ed.), Sex and Behavior, Nueva York, Wiley.

Davis, D. L. y R. G. Whitten (1987), "The Cross-Cultural Study of Human Sexuality”, Annual Review of Anthropology, vol. 16.

Davis, M. y E. Kennedy (1986), "Oral History and the Study of Sexuality in the Lesbian Community: Buffalo, Nueva York, 1940-1960”, Feminist Studies, vol. 12.

— y E. Kennedy (1989), "The Reproduction of Butchfem Roles: A Social Constructionist Approach", en C. Peiss y C. Simmons (eds.), Passion and Power: Sexuality in History, Filadelfia, Temple University Press.

D’Emiho, J. (1983), Sexual Politicis, Sexual Communities, Chicago, University of Chicago Press.

- y E. B. Freedman (1988), Intimate Matters: A Social History of Sexuality in America, Nueva York, Harper and Row.

Duberman, M. V., M. Vicinus y G. Chancey (eds.) (1989), Hidden from History: Reclaming the Gay and Lesbian Past, Nueva York, New American Library.

Duggan, L. (1990), "From Instincts to Politic: Writting the History of Sexuality in the U.S.", Joumal of Sex Research, vol. 27.

Ehrenreich, B. y D. English (1979), For Her Oum Good: 150 Years of Experts' Advise to Women, Nueva York, Doubleday.

Faderman, L. (1981), Surpassing the Love of Men. Nueva York.

Fausto-Sterling, A. (1985), Myths of Gender: Biological Theories about Women and Men, Nueva York, Basic Books.

Feldman, D. A. y T. M. Johnson (1986), The Social Dimension of .1ms: Method and Theory, Nueva York, Praegrer.

Fisher, L. (1980), "Relationships and Sexuality in Contexıs and Culture", en B. B. Wolman y J. Money (eds.), Handbook of Sexuality, Englewood Cliffs, Prentice-Hall. 
Ford, C. S., y F. A. Beach (1951), Patterns of Sexual Behavior, Nueva York, Harper and Row.

Foucault, M. (1978), The History of Sexuality, Nueva York, Pantheon.

Frayser, S. G. (1985), Varieties of Sexual Experience: An Anthropogical Perspective on Human Sexuality, New Haven, hRAF Press.

(1989), "Sexual and Reproductive Relationships: Cross-Cultural Evidence and Biosocial Implications", Medical Anthropology, vol. 11.

Fry, P. (1985), "Male Homosexuality and Spirit Possession in Brazil", Journal of Homosexuality, vol. 11.

Gagnon, J. H. y W. Simon (1973), Sexual Conduct: The Social Sources of Human Sexuality, Chicago, Aldine.

Gebhard, P. H. (1976), "Human Sexual Behavior: A Summary Statement", en C. Gordon y G. Jonhson (eds.), Readings in Human Sexuality: Contemporary Perspectives, Nueva York, Harper and Row.

Gerard, K. y G. Hekma (eds.) (1988), "The Pursuit of Sodomy: Male Homo sexual in Renaissance and Enlightenment Europe", Joumal of Homosexuality, vol. 16 (número especial).

Gever, M. (1989), "Pictures of Sickness: Stuart Marshall's Bright Eyes", en D. Crimp (ed.), ADs: Cultural Analysis, Cultural Activism, Cambrigde, мाт Press.

Gilman, S. L. (1988), Disease and Representation: Images of Illness since Madness to ADS, Ithaca, Cornell University Press.

- (1989), "AIDs and Syphilis: The Iconography of Disease", en D. Crimp (ed.), Ans: Cultural Analysis, Cultural Activism, Cambridge, MIT Press.

Goldenweiser, A. (1929), "Sex and Primitive Society", en V. F. Calverton y S. D. Schmalhausen (eds.), Sex in Civilization, Nueva York, Macaulay Company.

Goodenough, W. H. (1949), "Premarital Freedom in Truk: Theory and Practice", American Anthropologist, vol. 51.

Gordon, L. (1974), Woman's Body, Woman's Rigth: A Social History of Birth Control in America, Nueva York, Penguin.

Gorman, E. M. (1986), "The Aids Epidemic in San Francisco: Epidemiological and Anthropological Perspectives", en C. Jones, R. Stalls, y S. Gifford (eds.), Anthropology and Epidemiology, Dordrech, Reidel.

Gray, J. P. (1980), "Cross-Cultural Factors Associated with Sexual Foreplay", Joumal of Social Psychology, vol. 111.

Gregor, T. (1985), Anxious Pleasures: The Sexual Lives of an Amazonian People, Chicago, University of Chicago Press.

Grover, J. Z. (1989), "AiDs: Keywords", en D. Crimp (ed.), ADs: Cultural Analysis, Cultural Activism, Cambridge, miт Press.

Haraway, D. (1989), Primate Visions: Gender, Race and Nature in the World of Modern Science, Nueva York, Routlegde.

Harding, S. (1986), The Science Question in Feminism, Ithaca, Cornell University Press. 
__ Epistemology, Metaphysics, Methodology and Philosophy of Science, Dordrecht, Reidel.

Hawkeswood, W. G. (1990), "I'm a Black Young who just Happens to be Gay", trabajo presentado en la Convención Anual de la American Anthropological Asociation (mimeo.).

Heider, K. G. (1976), "Dani Sexuality: a Low-Energy System”, Man, vol. 11.

Herdt, G. (1981), Guardians of the Flutes, Nueva York, MacGraw-Hill.

(1984), "Semen Transaction in Sambia Culture", en G. Herdt (ed.), Ritualizaded Homosexuality in Melanesia, Berkeley, University of California Press.

(1987), The Sambia: Ritual and Gender in New Guinea, Nueva York, Holt, Rinehardt, Winston.

Honigman, J. J. (1954), “An Anthropological Approach to Sex”, Social Problems, vol. 2.

Howard, A. e I. Howard (1964), "Premarital Sex and Social Control among the Rotumans", American Anthropologist, vol. 66.

Hubbard, R., M. S. Henifin y B. Fried (1982), Biological Woman: The Convenient Myth, Cambrigde, Schenkman.

Irvine, J. (1990), Disorders of Desire, Filadelfia, Temple University Press.

Jordanova, L. J. (1989), Sexual Visions: Imogens of Gender in Science and Medicine between the Eigthteenth and Twentieth Centuries, Madison, University of Wisconsin Press.

Juhasz, A. (1990), "The Contained Threat: Women in Mainstream AIDs Documentary", Joumal of Sex Research, vol. 27.

Kane, S. (1990), "AIDS, Addiction and Condom Use: Sources of Sexual Risk for Heterosexual Women”, Joumal of Sex Research, vol. 27.

Kats, J. (1976), Gay American History, Nueva York, Crowell.

- (1983), Gay/Lesbian Almanac, Nueva York, Harper and Row.

(1990), "The Invention of Heterosexuality", Socialist Review, vol. 20.

Keller, E. F. (1984), Reflections on Gender and Science, New Haven, Yale University Press.

Kendrick, W. (1987), The Secret Museum, Nueva York, Viking.

Kinsey, A., W. Pomeroy y C. E. Martin (1948), Sexual Behavior in the Human Male, Filadelfia, Saunders.

- W. Pomeroy, C. E. Martin y R. H. Gebhard (1953), Sexual Behavior in the Human Female, Filadelfia, Saunders.

Lamphere, L. (1977), "Anthropology: A Review Essay", Signs, vol. 2.

Lessa, W. A. (1966), "Sexual Behavior", en Utilhi: A Design for Living, Nueva York, Holt, Rinehardt, Winston.

Levine, R. A. (1959), "Gusii Sex Offenses: A Study in Social Control", American Anthropologist, vol. 61.

Lowe, M., y R. Hubbard (1983), Women's Nature: Rationalizations of Inequality, Nueva York, Pergamon Press. 
Malinowski, B. (1941), The Sexual Life of Savages, Nueva York, Halcyon House. [1929]

Marshall, D. S. (1976), "Too much in Mangaia”, en C. Gordon y G. Jonhson (eds.), Readings in Human Sexuality: Contemporary Perspectives, Nueva York, Holt, Rinehardt, Winston.

—_ y R. C. Suggs (eds.) (1972), Human Sexual Behavior, Nueva Jersey, Englewood Cliffs/Prentice-Hall.

Marshall, P. A. y L. A. Bennett (eds.) (1990), "Culture and Behavior in the AIDs Epidemic", Medical Anthropology Quarterby, vol. 4 (número especial).

Mascia-Lees, F. E. (ed.) (1989), "Human Sexuality in Biocultural Perspective", Medical Anthropology, vol. 11.

—, F. D. Tierson y J. H. Relethford (1989), "Investigating the Biocultural Dimensions of Human Sexual Behavior”, Medical Anthropology, vol. 11.

McIntosh, M. (1968), "The Homosexual Role", Social Problems, vol. 16.

Mead, M. (1923), Coming in Age Samoa, Nueva York, Morrow.

(1961), "Cultural Determinants of Sexual Behaviors", en W. C. Young (ed.), Sex and Internal Secretions, Filadelfia, Williams and Wilkis.

Minturn, L., M. Grosse y S. Haider (1969), "Culture Patterning of Sexual Beliefs and Behavior", Ethnology, vol. 8.

Moore, H. L. (1988), Feminism and Anthropology, Minneapolis, University of Minnesota Press.

Murdock, G. P. (1949), "The Social Regulation of Sexual Behavior", en P. H. Hoch y J. Zubin (eds.), Psychosexual Develpment in Health and Disease, Nueva York, Grune and Stratton.

Murray, S. O. K. Payne (1989), "The Social Clasification of AIDs in the American Epidemiology", Medical Anthropology Quarterly, vol. 10.

Newton, E. (1979), Mother Camp: Female Impersonators in America, Chicago, University of Chicago Press.

(1984), "The Mythic Mannish Lesbian: Radclyffe Hall and the New Woman", Signs, vol. 9.

Padgug, R. A. (1979), "Sexual Matters: On Conceptualizing Sexuality in History", Radical History Review, vol. 20.

Parker, R. (1987), “Acquired Immunodeficiency Syndrome in Urban Brazil”, Medical Anthropology Quarterly, vol. 1.

(1991), Bodies, Pleasures, and Passions: Sexual Culture in Contemporary Brazil, Boston, Beacon Press.

Patton, C. (1985), Sex and Germs, Boston, South and Press.

Peiss, C. (1983), “'Carity girls' and City Pleasures: Historical Notes in Working Class Sexuality”, en A. Snitow, C. Stansell y S. Thompson (eds.), Powers of Desire, Nueva York, Monthly Review Press.

- (1986), Cheap Amusements: Working Women and Leisure in Turn-of theCentury Nueva York, Filadelfia, Temple University Press.

y C. Simmons (eds.) (1989), Passion and Power: Sexuality in History, Filadelfia, Temple University Press. 
Perper, T. (1989), "Theories and Observations on Sexual Selection and Female Choice in Human Beings", Medical Anthropology, vol. 11.

Pivar, D. (1972), Purity Crusade: Sexual Morality and Social Control 1868-1900, Connecticut, Greenwood Press.

Plummer, K. (ed.) (1981), The Making of the Modern Homosexual, Londres, Hutchinson.

(1982), "Symbolic Interactionism and Sexual Conduct: An Emergent Perspective", en M. Brake (ed.), Human Sexual Relations, Nueva York, Pantheon.

Rapp, R. (1979), "Anthropology: A Review Essay", Signs, vol. 4.

Reiter, R. (ed.) (1975), Toward an Anthropology of Women, Nueva York, Monthly Review Press.

Rosaldo, M. Z. y L. Lamphere (eds.) (1974), Women, Culture and Society, Stanford, Stanford University Press.

Rubin, G. (1975), "The Traffic in Women", en R. Reiter (ed.), Toward an Anthropology of Women, Nueva York, Monthly Review Press.

- (1979), "Introducción", en Renee Vivien, A Woman Appeared to Me, Weatherby Lake, Naiad Press.

- (1984), "Thinking Sex", en C. Vance, Pleasure and Danger: Exploring:Female Sexuality, Nueva York, Roudedge \& Kegan Paul.

Rupp, L. (1980), “'Imagine my Surprise': Women's Relationships in midTwentieth Century America", Frontiers, vol. 5.

Sahli, N. (1979), "Smashing: Women's Relationships before the Fall", Cyysalis, vol. 8.

Sayers, J. (1982), Biological Politics: Feminism and Antifeminism Perspectives, Nueva York, Tavistock Publications.

Schapera, I. (1941), Married Life in Africa Tribe, Nueva York, Sheridan House.

Schiebinger, L. (1989), The Minds Has no Sex: Women in the Origin of Modern Science, Cambridge, Harvard University Press.

Singer, M. et al. (1990), "Sida: The Economic, Social and Cultural Context of AIDs among Latinos", Medical Anthropology Quarterly, vol. 4.

Snitow, A., C. Stansell y S. Thompson (eds.) (1983), Powers of Desire, Nueva York, Monthly Review Press.

Stansell, C. (1986), City of Women: Sex and Class in Nueva York, Nueva York.

Suggs, R. C. (1966), Marquesan Sexual Behavior, Nueva York, Harcourt, Brace and World.

Tobach, E. y B. Rosoff (1978), Genes and Gender, 4 vols., Nueva York, Gordian Press.

Treichler, P. A. (1988), "AIDs, Gender and Biomedical Discourse: Current Contests for Meanings", en E. Fee y M. D. Box (eds.), The Burden of History, Berkeley, University of California Press. (1989), "AIDs, Homophobia and Biomedical Discourse: An Epidemic of Signification", en D. Crimp (ed.), AlDs: Cultural Analysis, Cultural Activism, Cambridge, MrT Press. 
Trimberger, E. K (1983), "Feminism, Men and Modern Love: Greenwich Village", en A. Snitow, C. Stansell y S. Thompson (eds.), Powers of Desire, Nueva York, Monthly Review Press.

Turner, C. F., H. G. Miller y L. E. Moses (eds.) (1989), ams, Sexual Behavior and Intravenous Drugs Use, Washington D.C., Comittee in Ans Research and the Behavioral, Social and Statistical Sciences, National Research Council, National Academy Press.

Vance, C. S. (1983), “Gender Systems, Ideology and Sex Research", en A. Snitow, C. Satnsell y S. Thompson (eds.), Powers of Desire, Nueva York, Monthly Review Press.

- (1984), Pleasure and Danger: Exploring Female Sexuality, Nueva York, Roudedge \&c Kegan Paul.

(1990), "Negotiating Sex and Gender in the Attorney General's Comission on Pornography", en F. Gingsburg y A. L. Tsing (eds.), Uncertain Terms: Negotiating Gender in America Culture, Boston, Beacon Press.

Vicinus, M. (1989), "The Wonder of Sex to which I Belong: The Historical Roots of the Modern Lesbian Identity", en D. Altmian, C. Vance, M. Vicinus y J. Weeks (eds.), Homosexuality, Which Homosexuality?, Amsterdam, An Dekker/Schorer.

Walkowitz, J. R. (1980), Prostitution and Victorian Society: Women, Class and the State, Cambridge, Cambridge University Press.

Watney, S. (1987), Policing Desire: Pornography, AlDs, and the Media, Minneapolis, University of Minnesota Press.

- (1989), "The Spectacle of Ans", en D. Crimp (ed.), Aws: Cultural Analysis, Cultural Activism, Cambridge, mrt Press.

Weeks, J. (1977), Coming Out: Homosexual Politics in Britain from the 19 th Century to the Present, Londres, Quarter Books.

- (1981), Sex, Politics and Ideology: the Regulation of Sex Since 1800, Nueva York, Longman.

(1986), Sexuality, Londres, Tavistock.

Whitehead, H. (1981), "The Bow and the Burden Strap: A New Look at Institutionalized Homosexuality in Native North America", en S. B. Ortner y H. Whitehead (eds.), Sexual Meanings, Cambridge, Cambridge University Press.

Williamson, J. (1989), "Every Virus Tells a Story: The Meanings of Hiv and Ans", en E. Carter y S. Watney (eds.), Taking Liberties: Ans and Cultural Politics, Londres, Serpent Tail. 